\title{
Increased levels of circulating adrenomedullin following treatment with TU-100 in patients with Crohn's disease
}

\author{
KEN KOMINATO, HIROSHI YAMASAKI, KEIICHI MITSUYAMA, HIDETOSHI TAKEDATSU, \\ SHINICHIRO YOSHIOKA, KOTARO KUWAKI, TEPPEI KOBAYASHI, \\ RYOSUKE YAMAUCHI, SHUHEI FUKUNAGA, OSAMU TSURUTA and TAKUJI TORIMURA \\ Inflammatory Bowel Disease Center, Division of Gastroenterology, Department of Medicine, \\ Kurume University School of Medicine, Kurume, Fukuoka 830-0011, Japan
}

Received July 31, 2015; Accepted June 27, 2016

DOI: $10.3892 / \mathrm{mmr} .2016 .5488$

\begin{abstract}
Daikenchuto (TU-100) is a traditional Japanese medicine that is widely used to treat intestinal symptoms. The mechanisms underlying it effects on the circulating levels of adrenomedullin (ADM) are of interest. In addition, the effect of TU-100 in the treatment of Crohn's disease (CD) in humans remains to be elucidated. The primary objective of the present study was to evaluate the effect of TU-100 on the circulating ADM levels in patients with active CD. An additional objective was to assess the effect of the drug on the disease activity and its potential side effects. In an open-label study, 10 patients with active CD received $15 \mathrm{~g}$ TU-100 per day for 8 consecutive weeks, and baseline anti-inflammatory therapy was continued. The pre- and post-treatment blood plasma levels of total ADM (t-ADM) and mature-ADM (m-ADM) were determined using enzyme-linked immunosorbent assays. The response of patients to the treatment was evaluated clinically using the International Organization for the Study of Inflammatory Bowel Diseases (IOIBD) score. The plasma levels of $t$-ADM $(16.4 \pm 1.1$ vs. $20.2 \pm 1.7 \mathrm{fmol} / \mathrm{ml}, \mathrm{P}=0.0218)$ and $\mathrm{m}-\mathrm{ADM}$ $(1.7 \pm 0.1$ vs. $2.2 \pm 0.1 \mathrm{fmol} / \mathrm{ml}, \mathrm{P}=0.0284)$ increased following 8 weeks of TU-100 treatment, compared with control. The IOIBD score of patients also improved, with a significant decrease in the score from $3.9 \pm 0.5$ at 0 weeks to $2.4 \pm 0.4$ at 8 weeks $(\mathrm{P}=0.0284)$. Out of the 10 components of the IOIBD scoring system, the scores for abdominal pain and tenderness, decreased significantly $(\mathrm{P}=0.014$ and $\mathrm{P}=0.046)$. Therefore, TU-100 was safe and well-tolerated by the patients that participated in the current study. The present study determined that the pharmacologic action of TU-100 is associated with changes in the circulating ADM levels and that treatment with TU-100
\end{abstract}

Correspondence to: Professor Keiichi Mitsuyama, Inflammatory Bowel Disease Center, Division of Gastroenterology, Department of Medicine, Kurume University School of Medicine, 67 Asahi-machi, Kurume, Fukuoka 830-0011, Japan

E-mail: ibd@med.kurume-u.ac.jp

Key words: Crohn's disease, daikenchuto, adrenomedullin may aid in the management of CD. These promising findings warrant further investigation in larger, multicenter studies.

\section{Introduction}

Crohn's disease (CD) is a chronic inflammatory disorder of the gastrointestinal tract. The precise cause remains unknown; however, genetic, immunological, infective and environmental factors have been proposed to contribute to its pathogenesis $(1,2)$.

Advances in the understanding of the pathogenesis of CD over the last decade have enabled the development of agents directed at rational therapeutic targets. Selective blocking of inflammatory cytokines through the introduction of novel biologicals, including anti-tumor necrosis factor- $\alpha$ agents, has yielded considerable clinical benefit (3). This approach validated the targeting of inflammatory cytokines as a strategy for treating ongoing disease; however, the long-term safety and efficacy of such therapeutic agents remains uncertain. Additionally, certain patients may not respond to this treatment, exhibit intolerance, or both. Thus, result in the requirement of an alternative therapeutic approach. The other treatments currently available include total parenteral nutrition and elemental diets, restriction of oral food intake and in severe cases, hospitalization. Therefore, a safer and more effective treatment method for CD is desirable.

Daikenchuto (TU-100) is a traditional Japanese herbal medicine (Kampo), which consists of a mixture of powdered extracts of dried Japanese pepper, processed ginger and ginseng radix, and maltose powder (4). TU-100 is a frequently prescribed Kampo medicine in Japan, particularly for the treatment of postoperative paralytic and adhesive ileus and ischemic intestinal disorders (5-8). Previous studies have demonstrated the effects of TU-100 on intestinal motility, adhesion, vasodilatation, inflammation and bacterial translocation (9-11). A previous study reported that the beneficial effect of TU-100 is primarily mediated by the increased release of adrenomedullin (ADM) from intestinal epithelial cells (12).

ADM, is a 52-amino-acid peptide, which was originally identified in human pheochromocytoma tissue. Subsequent studies have demonstrated that the ADM protein and mRNA are widely distributed in various tissues and organs (13-15). ADM 
promotes vasodilation, tissue repair and anti-inflammatory actions in the intestine. Exogenous administration of ADM has been demonstrated to exhibit therapeutic potential in various models of inflammatory disease, including colitis (16-18) and arthritis (19). In addition, it has been reported that TU-100 exerted beneficial effects in a mouse model of colitis through the induction of ADM release $(20,21)$.

On the basis of these previous studies, double-blind placebo controlled randomized clinical trials using TU-100 in patients with CD, postoperative paralytic ileus, functional constipation and irritable bowel syndrome have been conducted in Japan (funded by the Japanese Foundation For Multidisciplinary Treatment of Cancer: Identifier nos. JFMC39-0902, JFMC40-1001 and JFMC42-1002) and the United States (identifier nos. NCT00871325, NCT01139216, NCT01388933, and NCT01348152). However, to the best of our knowledge, the effects of TU-100 on the blood plasma levels of ADM in patients with $\mathrm{CD}$ have not been described previously.

The primary aim of the current study was to evaluate the efficacy of TU-100 treatment on the circulating ADM levels in patients with active $\mathrm{CD}$. Additional objectives included the assessment of the effect of the drug on the disease activity and its safety.

\section{Materials and methods}

Ethical approval. This project was performed in accordance with the principles laid out in the Declaration of Helsinki and with the approval of the Medical Ethics Committee of Kurume University Hospital (Kurume, Japan). Written informed consent was obtained from the participants.

TU-100 preparation. TU-100 was prepared as a dried powdered extract of Panacis Ginseng Radix (3.0 g), Zanthoxyli Fructus (2.0 g) and Zingiberis Siccatum Rhizoma (5.0 g), purchased from Tsumura \& Co., Ltd. (Tokyo, Japan).

Detection of ADM blood plasma levels. ADM occurs in two molecular forms, the active and mature ADM (m-ADM) form and the inactive and glycine-extended ADM (gly-ADM) form (22). Total ADM (t-ADM) is the sum of the levels of $\mathrm{m}-\mathrm{ADM}$ and gly-ADM. The present study determined the plasma levels of m-ADM and t-ADM by enzyme-linked immunosorbent assays (ELISA; Shionogi \& Co., Ltd., Tokyo, Japan). These assay systems use two monoclonal antibodies against human ADM, one that recognizes the ring structure of human $\mathrm{ADM}$ in each kit and another that recognizes the C-terminal sequence in the m-ADM kit or ADM- (25-36) in the t-AM kit. The assay determined human m-ADM or t-ADM by placing it between the two antibodies without plasma extraction. The detection limit was $2 \mathrm{fmol} / \mathrm{ml}$ for $\mathrm{t}-\mathrm{ADM}$ and $1 \mathrm{fmol} / \mathrm{ml}$ for $\mathrm{m}$-ADM. The plasma levels of calcitonin gene-related peptide (CGRP) were also determined by ELISA; the detection limit of this assay was $7.8 \mathrm{pg} / \mathrm{ml}$ (Bertin Pharma, Montigny le Bretonneux, France).

Patient selection and study design. An open-label, preliminary trial was conducted at Kurume University School of Medicine between September 2007 and June 2008. A total of 10 patients with active CD (3 men, 7 women; mean age,
Table I. Characteristics of 10 patients with active CD who completed 8 weeks of treatment with TU-100.

\begin{tabular}{lc} 
Total number of patients & 10 \\
Mean age \pm SE (years) & $37.3 \pm 15.5$ \\
Number of males & 3 \\
Mean duration of disease since & $11.7 \pm 13.2$ \\
diagnosis \pm SE (years) & $3.9 \pm 0.55$ \\
Mean baseline IOIBD \pm SE & $1.2 \pm 0.3$ \\
Mean baseline CRP \pm SE (mg/dl) & \\
Location of disease, $\mathrm{n}$ & 2 \\
Ilial & 1 \\
Colonic & 7 \\
Ileocolonic & \\
Disease behavior, $\mathrm{n}$ & 1 \\
Inflammatory & 6 \\
Stricturing & 3 \\
Penetrating & 6 \\
Previous intestinal resection, $\mathrm{n}$ & \\
Concomitant medication, $\mathrm{n}(\%)$ & $10(100)$ \\
Mesalamine & $4(40)$ \\
Corticosteroids & $0(0)$ \\
Antibiotics & $1(10)$ \\
Azathioprine & $2(20)$ \\
Anti-tumor necrosis factor & $7(70)$ \\
Nutritional therapy & \\
\hline
\end{tabular}

$\mathrm{CD}$, Crohn's disease; IOIBD, International Organization for the Study of Inflammatory Bowel Diseases; CRP, C-reactive protein; SD, standard deviation.

37.3 years; mean duration of $\mathrm{CD}, 11.7$ years) participated in this study (Table I). The diagnosis of CD was based on characteristic clinical, endoscopic, radiological and histological features. The patients had been unresponsive or intolerant to the standard treatment methods for CD for at least 4 weeks. All baseline anti-inflammatory therapies were continued throughout the current study (Table I). No dietary alterations were made after the patients entered the study.

Patients received $15 \mathrm{~g}$ TU-100 (5 g three times a day administered orally) per day for 8 consecutive weeks. Plasma levels of m-ADM, t-ADM and CGRP were determined before and after the 8-week administration.

The response to the treatment was evaluated biweekly using the routine laboratory variables, including serum hemoglobin and C-reactive protein (CRP) and the International Organization for the Study of Inflammatory Bowel Disease (IOIBD) score (23). The IOIBD score is based on 10 components, specifically the presence/absence of: i) Abdominal pain, ii) bowel evacuation 6 times or more per day, iii) anal lesion, iv) fistula, v) complication, vi) abdominal tumor, vii) body weight decrease, viii) fever of $38^{\circ} \mathrm{C}$ or higher, ix) abdominal tenderness and $\mathrm{x}$ ) hemoglobin level of $10 \mathrm{~g} / \mathrm{dl}$ or lower. Presence of each of these components is assigned 1 point, and the total number of points is expressed as the IOIBD score of the patients. 
Statistical analysis. Results are expressed as the mean \pm standard error. All the statistical analyses were performed using SPSS version 12.0.2 J (SPSS Inc., Chicago, IL, USA). Data were compared using Wilcoxon signed-rank tests or paired Student's t tests. Spearman's rank correlation test was used where appropriate. $\mathrm{P}<0.05$ was considered to indicate a statistically significant difference.

\section{Results}

Effect of TU-100 on the circulating ADM levels. The t-ADM and m-ADM levels in blood plasma of patients at the beginning and the end of treatment with TU-100 are presented in Fig. 1. Detectable levels of ADM were identified in all of the plasma samples. Following 8 weeks of treatment with TU-100, the patients had significantly elevated levels of plasma t-ADM $(20.2 \pm 1.7 \mathrm{fmol} / \mathrm{ml})$ compared with levels prior to treatment $(16.4 \pm 1.1 \mathrm{fmol} / \mathrm{ml} ; \mathrm{P}=0.0218)$ and plasma m-ADM $(2.2 \pm 0.1 \mathrm{vs}$. $1.7 \pm 0.1 \mathrm{fmol} / \mathrm{ml} ; \mathrm{P}=0.0284)$. Simultaneous determinations revealed the absence of detectable CGRP in the plasma in the majority of subjects.

Effect of TU-100 on the disease activity of $C D$. The changes in the activity of the disease during TU-100 treatment are presented in Fig. 2. The IOIBD score significantly improved over time, with a decrease in the score from $3.9 \pm 0.5$ to $2.4 \pm 0.4$ $(\mathrm{P}=0.0284)$. A favorable trend was observed in the serum CRP concentration; however, no significant difference was identified. The effect of TU-100 treatment on the components of the IOIBD scoring system was also examined (Fig. 3). Out of the 10 components, the scores for abdominal pain and tenderness were significantly decreased after 8 weeks when compared with the scores at the start of the study $(\mathrm{P}=0.014$ and $\mathrm{P}=0.046$, respectively). No significant differences were identified for the remaining components.

Correlation between the circulating levels of ADM and the disease activity. As presented in Fig. 4, no significant association was observed between the plasma concentrations of ADM and the IOIBD scores or serum CRP levels.

Side effects. TU-100 was well tolerated in all 10 patients enrolled in the present study. The patients felt well and did not experience any side effects. No febrile or allergic reactions were observed. No changes were encountered in the routine blood biochemical parameters, including liver and renal function tests, during/after TU-100 treatment. Additionally, no adverse events related to TU-100 were observed.

\section{Discussion}

To the best of our knowledge, the present study was the first to evaluate the efficacy, circulating levels of ADM, disease activity and safety of TU-100 in patients with CD.

Due to the heterogeneous nature of TU-100, the underlying mechanism of its therapeutic effect has not been fully elucidated. However, observations in animal models indicate that the effect of TU-100 may be attributed to the induction of ADM release. TU-100 also improves colonic injury by enhancing endogenous ADM release (20). Additionally,

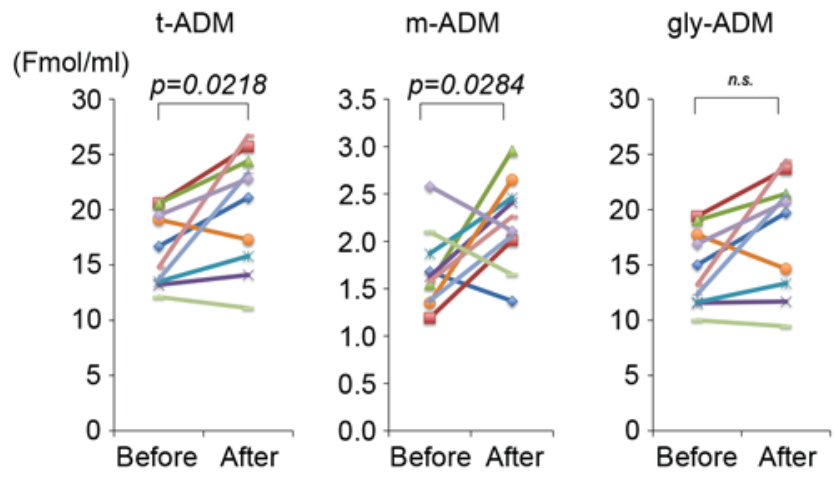

Figure 1. Plasma concentrations of t-ADM, m-ADM and gly-ADM of the 10 patients with Crohn's disease before and after treatment with TU-100 for 8 weeks. The plasma concentrations of t-ADM and m-ADM were determined using enzyme-linked immunosorbent assays, whereas those of gly-ADM were calculated by the subtraction of the plasma m-ADM concentrations from the plasma t-ADM concentrations. t-ADM, total ADM; m-ADM, mature ADM; gly-ADM, glycine-extended ADM.
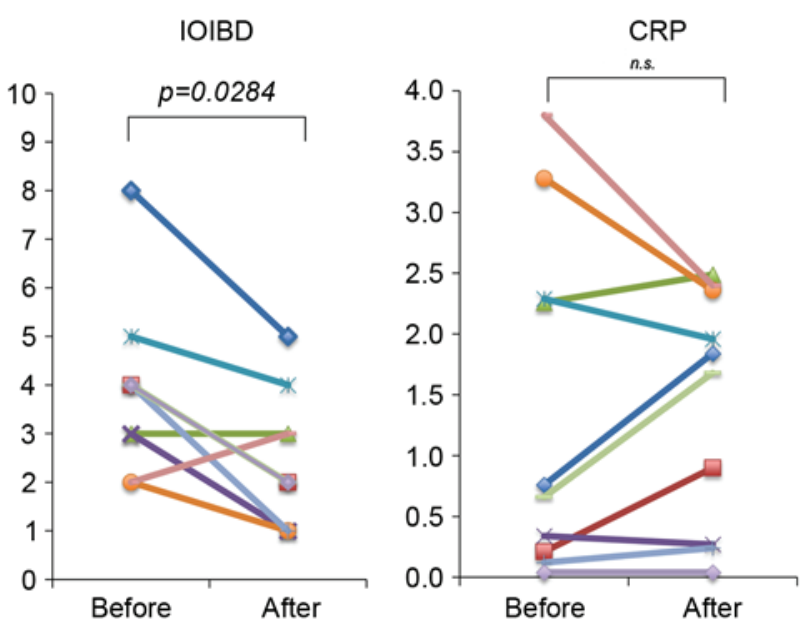

Figure 2. IOIBD scores and serum CRP levels of the 10 patients with Crohn's disease before and after treatment with TU-100 for 8 weeks. IOIBD, International Organization for the Study of Inflammatory Bowel Disease; CRP, C-reactive protein.

the beneficial effect of TU-100 was reduced by an ADM inhibitor (11). Systemically administered ADM had a similar beneficial effect on colonic injury (16-19).

Therefore, there was considerable interest in determining whether TU-100 promoted ADM release in patients with CD. The present study determined that TU-100 treatment induced the release of the active form of ADM, which possibly resulted in a significant improvement of the disease activity. These findings suggest that the efficacy of TU-100 against CD may be contributed to the induction of ADM release. To the best of our knowledge, the present study was the first to determine the circulating levels of ADM in blood plasma during TU-100 treatment in humans. At present, the current findings on the clinical response of patients with CD to TU-100 are preliminary. However, as TU-100 has been observed to promote ADM release in human subjects is encouraging and further clinical investigations should be performed.

The mechanisms underlying the effect of TU-100 on the release of ADM remain to be fully elucidated. TU-100 

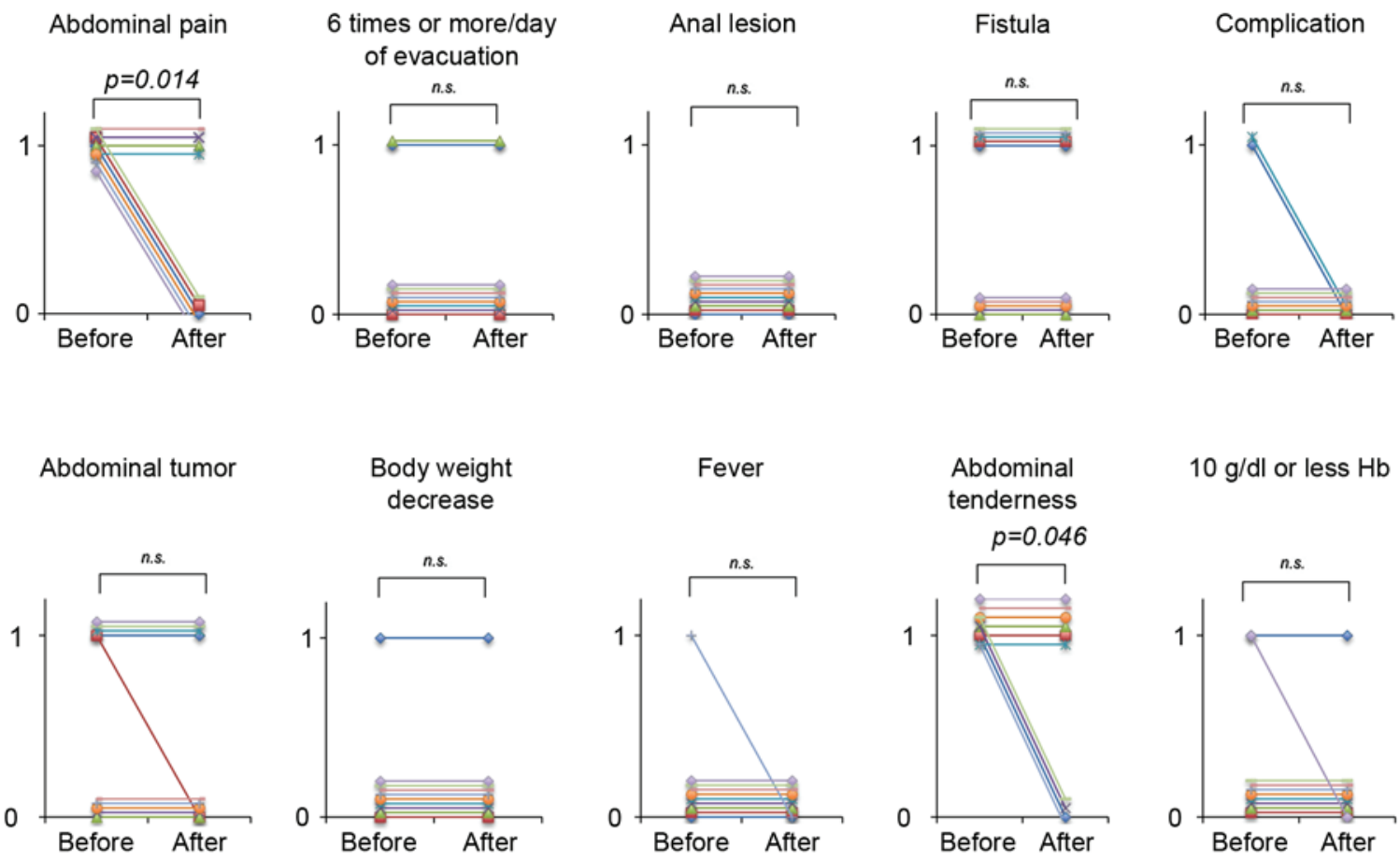

$10 \mathrm{~g} / \mathrm{dl}$ or less $\mathrm{Hb}$

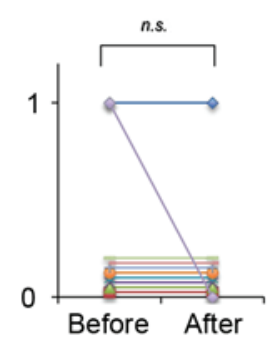

Figure 3. Score for each item of the IOIBD score in the 10 patients with Crohn's disease before and after treatment with TU-100 for 8 weeks. IOIBD, International Organization for the Study of Inflammatory Bowel Disease; Hb, hemoglobin.
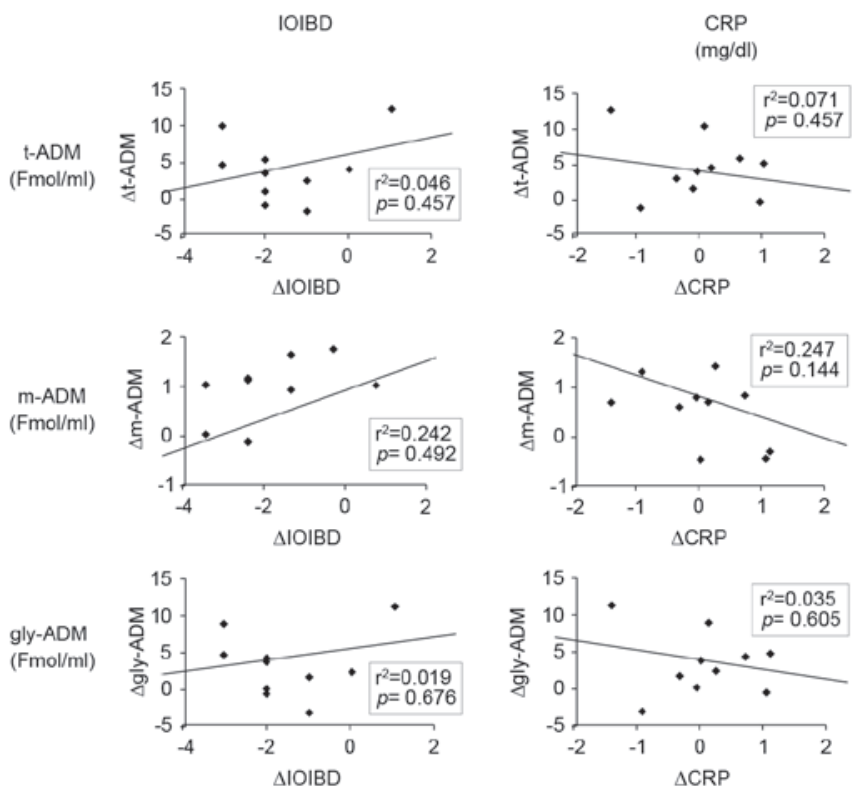

Figure 4. Correlation between the plasma concentrations of t-ADM, m-ADM and gly-ADM, and the IOIBD score or serum CRP. IOIBD, International Organization for the Study of Inflammatory Bowel Disease; CRP, C-reactive protein; t-ADM, total ADM; m-ADM, mature ADM; gly-ADM, glycine-extended ADM.

components, including 6-shogaol and hydroxy- $\alpha$-sanshool, contain an activator of the Transient Receptor Potential (TRP)-channels (4). Kono et al (24) demonstrated that TU-100 and its component 6-shogaol increase the intestinal blood flow via enhancement of ADM release mediated by the stimulation of TRP ankyrin 1 in intestinal epithelial cells (24). It possible that a component of TU-100, such as 6-shogaol, is responsible for ADM release through TRP channel stimulation.
Determination of the signaling pathways involved in the TRP channel-ADM axis is an important area for further investigation.

No direct correlation was observed between the circulating levels of ADM and the IOIBD scores during TU-100 treatment. Therefore, an additional unrecognized peptide may be involved in the response to TU-100 treatment, in addition to ADM. Further investigation is required to elucidate the precise mechanisms underlying the actions of TU-100 action in human patients with $\mathrm{CD}$.

The primary advantage of TU-100 is that it appears to lack any side effects or toxicity (4). In particular, TU-100 led to no allergic reactions or any significant changes in blood biochemical parameters, including liver and renal function tests, during the study period. Standard therapy for CD consists of drugs that primarily act on the intestinal immune-component cells, including lymphocytes and macrophages to reduce the release of various inflammatory mediators (3). However, the preventive action of TU-100 differs from these anti-inflammatory approaches. Combined therapy of TU-100 and standard therapeutic agents is of interest and warrants evaluation.

The findings of the current study suggest that treating CD using ADM may be a novel approach to treat CD (16-19). In addition, ADM, particularly its active form, are rapidly cleared from the blood, which decreases the systemic effects (25). By contrast, TU-100 appears to affect the endogenous ADM system not systemically, but locally and in a sustained manner. Such sustained and localized increase of ADM induced by TU-100 may be advantageous, as it may lead to the biological effect of ADM being primarily confined to the disease sites (12).

A potential limitation of the current study is that it included a small number of patients. The patients were monitored for 
8 weeks subsequent to the administration of the drug; however, a trial with a greater number of patients and longer-term follow-up period is required.

In conclusion, the present study provides novel evidence to suggest that TU-100 may be an effective treatment for $\mathrm{CD}$, and exerts this effect at least in part through inducing the release of ADM. Additionally, no significant side effects or toxicity were observed during this trial. Based on these encouraging preliminary results, a larger, multicenter randomized controlled clinical trial of this therapeutic agent should be conducted for patients with CD.

\section{Acknowledgements}

The present study was supported in part by a Grant-in-Aid from the Japanese Ministry of Health and Welfare. The abstract was presented at the UEG Week 2013 Poster Presentations October 14 2013, and published in United European Gastroenterol J 1, A135-A587 (1 Suppl): 2013.

\section{References}

1. Neurath MF and Finotto $S$ : The many roads to inflammatory bowel diseases. Immunity 25: 189-191, 2006.

2. Strober W, Fuss I and Mannon P: The fundamental basis of inflammatory bowel disease. J Clin Invest 117: 514-521, 2007.

3. Vermeire S, Ferrante M and Rutgeerts P: Recent advances: Personalised use of current Crohn's disease therapeutic options. Gut 62: 1511-1515, 2013.

4. Kono T, Kanematsu T and Kitajima M: Exodus of Kampo, traditional Japanese medicine, from the complementary and alternative medicines: Is it time yet? Surgery 146: 837-840, 2009.

5. Endo S, Nishida T, Nishikawa K, Nakajima K, Hasegawa J, Kitagawa T, Ito T and Matsuda H: Dai-kenchu-to, a Chinese herbal medicine, improves stasis of patients with total gastrectomy and jejunal pouch interposition. Am J Surg 192: 9-13, 2006.

6. Itoh T, Yamakawa J, Mai M, Yamaguchi $\mathrm{N}$ and Kanda T: The effect of the herbal medicine dai-kenchu-to on post-operative ileus. J Int Med Res 30: 428-432, 2002.

7. Iwai N, Kume Y, Kimura O, Ono S, Aoi S and Tsuda T: Effects of herbal medicine Dai-Kenchu-to on anorectal function in children with severe constipation. Eur J Pediatr Surg 17: 115-118, 2007.

8. Suehiro T, Matsumata T, Shikada Y and Sugimachi K: The effect of the herbal medicines dai-kenchu-to and keishi-bukuryo-gan on bowel movement after colorectal surgery. Hepatogastroenterology 52: 97-100, 2005.

9. Tokita Y, Satoh K, Sakaguchi M, Endoh Y, Mori I, Yuzurihara M, Sakakibara I, Kase Y, Takeda S and Sasaki H: The preventive effect of Daikenchuto on postoperative adhesion-induced intestinal obstruction in rats. Inflammopharmacology 15: 65-66, 2007.

10. Murata P, Kase Y, Ishige A, Sasaki H, Kurosawa S and Nakamura T: The herbal medicine Dai-kenchu-to and one of its active components [6]-shogaol increase intestinal blood flow in rats. Life Sci 70: 2061-2070, 2002.
11. Kono T, Koseki T, Chiba S, Ebisawa Y, Chisato N, Iwamoto J and Kasai S: Colonic vascular conductance increased by Daikenchuto via calcitonin gene-related peptide and receptor-activity modifying protein 1. J Surg Res 150: 78-84, 2008.

12. Kono T, Kaneko A, Hira Y, Suzuki T, Chisato N, Ohtake N, Miura N and Watanabe T: Anti-colitis and -adhesion effects of daikenchuto via endogenous adrenomedullin enhancement in Crohn's disease mouse model. J Crohns Colitis 4: 161-170, 2010.

13. Eto T: A review of the biological properties and clinical implications of adrenomedullin and proadrenomedullin N-terminal 20 peptide (PAMP), hypotensive and vasodilating peptides. Peptides 22: 1693-1711, 2001

14. Julián M, Cacho M, García MA, Martín-Santamaría S, de Pascual-Teresa B, Ramos A, Martínez A and Cuttitta F: Adrenomedullin: A new target for the design of small molecule modulators with promising pharmacological activities. Eur J Med Chem 40: 737-750, 2005.

15. Marutsuka K, Nawa Y, Asada Y, Hara S, Kitamura K, Eto T and Sumiyoshi A: Adrenomedullin and proadrenomudullin $\mathrm{N}$-terminal 20 peptide (PAMP) are present in human colonic epithelia and exert an antimicrobial effect. Exp Physiol 86: 543-545, 2001.

16. Gonzalez-Rey E, Fernandez-Martin A, Chorny A and Delgado M: Therapeutic effect of urocortin and adrenomedullin in a murine model of Crohn's disease. Gut 55: 824-832, 2006.

17. Talero E, Sánchez-Fidalgo S, de la Lastra CA, Illanes M, Calvo JR and Motilva V: Acute and chronic responses associated with adrenomedullin administration in experimental colitis. Peptides 29: 2001-2012, 2008.

18. Ashizuka S, Ishikawa N, Kato J, Yamaga J, Inatsu H, Eto T and Kitamura K: Effect of adrenomedullin administration on acetic acid-induced colitis in rats. Peptides 26: 2610-2615, 2005.

19. Gonzalez-Rey E, Chorny A, O'Valle F and Delgado M: Adrenomedullin protects from experimental arthritis by down-regulating inflammation and Th1 response and inducing regulatory T cells. Am J Pathol 170: 263-271, 2007.

20. Kono T, Omiya Y, Hira Y, Kaneko A, Chiba S, Suzuki T, Noguchi M and Watanabe T: Daikenchuto (TU-100) ameliorates colon microvascular dysfunction via endogenous adrenomedullin in Crohn's disease rat model. J Gastroenterol 46: 1187-1196, 2011.

21. Kaneko A, Kono T, Miura N, Tsuchiya N and Yamamoto M: Preventive effect of TU-100 on a Type-2 model of colitis in mice: Possible involvement of enhancing adrenomedullin in intestinal epithelial cells. Gastroenterol Res Pract 2013: 384057, 2013.

22. Nishikimi T, Shibasaki I, Iida H, Asakawa H, Matsushita Y, Mori H, Mochizuki Y, Okamura Y, Horinaka S, Kangawa K, et al: Molecular forms of adrenomedullin in pericardial fluid and plasma in patients with ischaemic heart disease. Clin Sci (Lond) 102: 669-677, 2002

23. Myren J, Bouchier IA, Watkinson G, Softley A, Clamp SE and de Dombal FT: The O.M.G.E. multinational inflammatory bowel disease survey 1976-1982. A further report on 2,657 cases. Scand J Gastroenterol Suppl 95: 1-27, 1984.

24. Kono T, Kaneko A, Omiya Y, Ohbuchi K, Ohno N and Yamamoto M: Epithelial transient receptor potential ankyrin 1 (TRPA1)-dependent adrenomedullin upregulates blood flow in rat small intestine. Am J Physiol Gastrointest Liver Physiol 304: G428-G436, 2013.

25. Nishikimi T, Matsuoka H, Shimada K, Matsuo H and Kangawa K: Production and clearance sites of two molecular forms of adrenomedullin in human plasma. Am J Hypertens 13: 1032-1034, 2000 . 